\title{
Current Degradation and Coupling Losses in Ring Shaped Wires due to Applied Magnetic Field Changes
}

\author{
E.M.J. Niessen, A. Nijhuis, E.J. Oetsen and L.J.M. van de Klundert \\ University of Twente, Dept. of Applied Physics \\ P.O. Box 217, 7500 AE Enschede, The Netherlands
}

\begin{abstract}
The stationary electric field and current distribution in a ring shaped wire subject to both parallel and perpendicular applied magnetic fields is calculated. The time derivative of the parallel field is limited for the centre of the wire to be unsaturated. The transient response of an infinitely long wire due to a parallel applied magnetic field $B^{A}$ shows that $B^{A}$ itself is limited for the centre of the wire to be unsaturated. The measured V-I characteristic of a ring shaped wire is linear dependent on the frequency of the applied field but non linear dependent on the amplitude. This means that the stationary analysis is not complete and transient effects must be considered.
\end{abstract}

\section{INTRODUCTION}

The transport current in a multifilamentary wire is not only limited by the magnetic field dependence of the critical current but also by the induced currents resulting from the time dependence of the magnetic field. This transport current degradation can be studied using a ring shaped multifilamentary wire subject to constant and time dependent applied magnetic fields.

A theoretical description of the problem is difficult because the geometry of the ring, the applied magnetic field and the internal structure of the twisted superconducting wire is complicated. We first consider the geometry and the applied magnetic field. The circular wire with radius $a$ is bent in a ring shape with radius $R_{0}$, with $a \ll R_{0}$. It is possible to translate the problem into a problem describing a straight wire with adapted applied magnetic field which takes into account the bending of the wire [1]. This can be done for twistlength $L_{p}$ small compared to the radius of the ring $R_{0}$. We choose the centre of the wire to coincide with the origin of the cylindrical $r, \phi, z$ coordinate system and the axis parallel to the $z$ coordinate. The adapted applied magnetic field then becomes a periodic function of $z$. This field can be expanded in a Fourier series with respect to the $z$ component and the electic field can be calculated for every Fourier term and addition of the terms gives the total electric field. This approach is valid as long as the problem is linear, i.e. no saturation occurs in the wire.

First the solution for the periodic applied magnetic field is calculated assuming $\dot{B}^{I}=0$ : the time derivative of the induced magnetic field is zero. Also transient response to a parallel applied magnetic field on a infinitely long wire is investigated. Furthermore, the experimental set-up is described and first results are presented.

\section{PERIODIC APPLIED MAGNETIC FIELD}

In the analysis we consider for the applied field only the lowest Fourier mode. Then the applied magnetic field is:

$$
\begin{aligned}
& \dot{B_{x}^{A}}=\dot{B_{\perp w}^{A}} \sin \mu z+\dot{B_{\| w}^{A}} \frac{\mu r}{2} \cos \phi \sin \mu z+O\left(r^{2}\right), \\
& \dot{B_{y}^{A}}=\dot{B_{\| w}^{A}} \frac{\mu r}{2} \sin \phi \sin \mu z+O\left(r^{2}\right),
\end{aligned}
$$

Manuscript received June 25, 1991. This work was partly supported by NET contract.

$$
\dot{B_{z}^{A}}=\dot{B_{\| w}^{A}} \cos \mu z+\dot{B_{\perp w}^{A}} \mu r \cos \phi \cos \mu z+O\left(r^{2}\right),
$$

with $\mu=2 \pi / L$ and $L=2 \pi R_{0}$. The subscripts $\| w$ and $\perp w$ stand for parallel and perpendicular to the wire. Then:

$$
\begin{aligned}
\dot{B_{r}^{A}} & =\dot{B_{\perp w}^{A}} \cos \phi \sin \mu z+\dot{B_{\| w}^{A}} \frac{\mu r}{2} \sin \mu z+O\left(r^{2}\right) \\
\text { and } \dot{B_{\phi}^{A}} & =-B_{\perp w}^{\dot{A}} \sin \phi \sin \mu z+O\left(r^{2}\right) .
\end{aligned}
$$

The dominant terms of the applied field are the $x$ and $z$ component accounting for the perpendicular and parallel field terms. The terms which are a function of $r$ arise because the applied field must satisfy $\vec{\nabla} \cdot \overrightarrow{B^{A}}=0$ and $\vec{\nabla} \times \overrightarrow{B^{A}}=\overrightarrow{0}$ on the wire.

The internal structure of the wire is described using Carr's continuum model [2] and the critical state model describing the filaments $[3,4]$. In the unsaturated regions, the electric field parallel to the filaments $E_{\|}=\cos \psi E_{z}+\sin \psi E_{\phi}$ is equal zero. Here $\tan \psi=\beta r$, with $\beta=2 \pi L_{p}^{-1}$. This gives: $E_{z}=-\beta r E_{\phi}$. Substitution in $\vec{\nabla} \times \vec{E}=-\dot{\overrightarrow{B^{A}}}$ results in:

$$
\left(\partial_{z}+\beta \partial_{\phi}\right) E_{\phi}=\dot{B_{r}^{A}} \text { and }\left(\partial_{z}+\beta \partial_{\phi}\right) E_{r}=-\dot{B_{\phi}^{A}}+\beta r \dot{B_{z}^{A}} .
$$

Here the time derivative of the induced magnetic field $\dot{B}^{I}$ is considered to be zero, resulting in a time-independent problem. Conservation of current $(\vec{\nabla} \cdot \vec{j}=0)$ is written as:

$$
\begin{gathered}
\partial_{z} j_{\diamond}+\beta \partial_{\phi} j_{s}+\frac{\sigma_{\perp}}{r}\left(\partial_{r} r E_{r}+\partial_{\phi} E_{\phi}\right)-\beta r \sigma_{\perp} \partial_{z} E_{\phi}=0 \\
\text { using }\left(j_{\phi}, j_{z}\right)=\left(\beta r j_{\bullet}+\sigma_{\perp} E_{\phi}, j_{\diamond}-\beta r \sigma_{\perp} E_{\phi}\right)
\end{gathered}
$$

$j_{s}=\cos \psi j_{\|}$and $\sigma_{\perp}=\sigma(1-\eta) /(1+\eta) . \sigma$ is the conductivity of the normal conducting matrix material and $\eta$ is the fraction of superconducting material. For periodic boundary conditions on $z=0$ and $z=L$, the solution, first order accurate in $r$, is:

$$
\begin{aligned}
E_{\phi}= & \dot{B_{\perp w}^{A}} c_{1}(\sin \phi \sin \mu z+q \cos \phi \cos \mu z)-\dot{B_{\| w}^{A}} \frac{r}{2} \cos \mu z \quad(9) \\
E_{r}= & \dot{B_{\perp w}^{A}} c_{1}(q \sin \phi \cos \mu z-\cos \phi \sin \mu z)+\dot{B_{\| w}^{A}} \frac{r}{q} \sin \mu z(10) \\
j_{s}= & \frac{L_{p} \sigma_{\perp}}{\pi q^{2}} \dot{B_{\| w}^{A}} \cos \mu z-\frac{q\left(1+q^{2}\right)}{1-q^{2}} c_{1} \sigma_{\perp} \beta r \dot{B}_{\perp w}^{A} \cos \phi \cos \mu z \\
& -\frac{q^{2}}{1-q^{2}} 2 c_{1} \sigma_{\perp} \beta r \dot{\perp}_{\perp w}^{A} \sin \phi \sin \mu z
\end{aligned}
$$

with $c_{1}=1 /\left(\beta\left(1-q^{2}\right)\right)$ and $q=L_{p} / L$. With the approximations the coupling loss power density in the unsaturated case reads:

$$
\begin{aligned}
P / \mathrm{Vol} & =\frac{\sigma_{\perp}}{\mathrm{Vol}} \int_{0}^{L} \int_{-\pi}^{\pi} \int_{0}^{a}\left(E_{\perp}^{2}+E_{r}^{2}\right) r d z d \phi d r\left[W / m^{3}\right], \\
& =\frac{\left(4+q^{2}\right)}{16 q^{2}} a^{2}\left(B_{\| w}^{\dot{A}}\right)^{2} \sigma_{\perp}+\frac{1+q^{2}}{2\left(1-q^{2}\right)^{2}} \frac{L_{p}^{2}}{4 \pi^{2}}\left(B_{\perp w}^{\dot{A}}\right)^{2} \sigma_{\perp} .
\end{aligned}
$$

Here $\mathrm{Vol}=\pi L a^{2}$ and $E_{\perp}=E_{\phi}$. These formulae are only valid in the limit $q \rightarrow 0$ [1]. Note that the expressions nicely seperate the contribution of the parallel field component from that of the perpendicular one. We can see that the singular behaviour for $q \rightarrow 0$ is due to the field component parallel to the wire. The singularity of $E_{r}$ and the currents can be understood as follows: a $\dot{B}^{A}$ component parallel to the axis of a twisted wire causes a bulk 
712

current to flow in the direction of the magnetic field and a surface current in the opposite direction, both currents proportional to the length of the wire placed in this field. Due to the twisting of the wire, the penetrated flux is linear proportional to the length of the wire in the parallel field. At $\mu z= \pm \frac{\pi}{2}$ the currents are very small compared to the values for $\mu z=0$, which means that the current loops have closed via the $r$ direction. This causes high values of $E_{r}$ in these closing regions.

The maximum value of $\left|\dot{B_{\mid w}^{A}}\right|$ for which the centre of the wire is unsaturated is given for small values of $q$ by:

$$
\left|\dot{B_{|| w}}\right|<\frac{\eta j_{c} L_{p}}{4 \pi R_{0}^{2} \sigma_{\perp}} .
$$

Notice that $|\dot{B}|$ must be very small for large values of $R_{0}$ for the centre of the wire to be unsaturated. In the next section where we consider time dependent effects we will see that not only $\left|\dot{B_{i \mid w}^{A}}\right|$ but also $\left|B_{\| \mid w}^{A}\right|$ is a limiting factor.

\section{TRANSIENT RESPONSE TO PARALLEL FIELDS}

In the previous section the solution of the Maxwell equations has been studied assuming $\dot{B}^{I}=0$. The results however, may be obtained after a long time since the development of this solution takes some time. This problem has been studied previously by Turck, Ries and others $[5,6]$. The time dependent effects consist of two parts: the time dependency of the superconducting and of the normal currents. In the analytical approach we assume no normal currents present $\left(\sigma_{\perp}=0\right)$ so only the time dependency with respect to the superconducting currents is considered.

We consider an infinitely long twisted wire in a rotational symmetric parallel applied field:

$$
\left(B_{\tau}^{A}, B_{\phi}^{A}, B_{z}^{A}\right)=(0,0, \alpha t),
$$

with $\alpha$ a constant. The Maxwell's equations read:

$$
\begin{aligned}
\partial_{r} E_{z} & =\dot{B}_{\phi}, \\
\frac{1}{r} \partial_{r} r E_{\phi} & =-\dot{B}_{z}, \\
-\partial_{r} B_{z} & =\mu_{0} j_{\phi} \\
\text { and } \frac{1}{r} \partial_{r} r B_{\phi} & =\mu_{0} j_{z},
\end{aligned}
$$

with $\vec{B}=\overrightarrow{B^{A}}+\overrightarrow{B^{I}}$. Using $\vec{\nabla} \cdot \vec{B}=0$ and $(\vec{\nabla} \times \vec{E})_{r}=-\dot{B}_{r}$ we obtain $B_{r}=0$ and using $(\vec{\nabla} \times \vec{B})_{r}=\mu_{0} j_{r}=\mu_{0} \sigma_{\perp} E_{r}$ we obtain $E_{r}=0$ : no current exchange takes place between the inner and outer layers. The boundary and initial conditions read:

$$
\begin{aligned}
E_{\phi}(r=0, t)=B_{\phi}(r=0, t)=B_{\phi}(r=a, t) & =0, \\
B_{z}(r=a, t) & =B_{z}^{A} \\
\text { and } \vec{E}(t \leq 0)=\vec{B}(t \leq 0) & =0 .
\end{aligned}
$$

At $t=0$ the wire is completely unsaturated. As $t$ increases the unsaturated state may remain for some $r$ regions, whereas in others saturation will occur. Using the Maxwell's equations and $(8), E_{\phi}$ in the unsaturated regions is given by:

$$
\partial_{r} \frac{1}{r}\left(1+\beta^{2} r^{2}\right) \partial_{r} r E_{\phi}=\left(1+\beta^{2} r^{2}\right) \mu_{0} \sigma_{\perp} \dot{E}_{\phi} .
$$

For $\sigma_{\perp}=0$ the solution is:

$$
\begin{aligned}
& \left(E_{r}, E_{\phi}, E_{z}\right)=\left(0, \frac{C}{2 \beta^{2} r} \ln \left(1+\beta^{2} r^{2}\right),-\beta r E_{\phi}\right), \\
& \left(B_{r}, B_{\phi}, B_{z}\right)=\frac{-C t}{1+\beta^{2} r^{2}}(0, \beta r, 1)
\end{aligned}
$$

$$
\text { and } \mu_{0} j_{,}=\frac{-2 C t \beta}{\left(1+\beta^{2} r^{2}\right)^{2}} \text {. }
$$

Since $B_{\phi}^{A}(r=a)=0$ i.e. there is no applied current, the current in the outer region close to the surface will oppose that in the inner region and also will be saturated $\left(j_{\|}=-j_{c}\right)$ if $\alpha>0$. We can confine this return current in a superconducting surface current at $r=a$ :

$$
\frac{J_{\phi}}{\beta a}=J_{z}=-\frac{1}{a} \int_{0}^{a} j_{z} r d r
$$

Then in the unsaturated region $B_{z}(a)=\alpha t+\mu_{0} J_{\phi}=-C t /(1+$ $\left.\beta^{2} a^{2}\right)$ and $B_{\phi}(a)=-\mu_{0} J_{z}=-\beta a C t /\left(1+\beta^{2} a^{2}\right)$. Eliminating $J_{s}$ gives: $C=-\alpha$. The saturation in the inner region thus will start if: $j_{s}=\eta j_{c} \cos \psi$, so at $r=0$ and $B_{z}^{A}=\alpha t=\mu_{0} \eta j_{c} L_{p} /(4 \pi)$ and gradually fill the whole wire.

The numerical solution is calculated taking all time dependent effects and the saturation of filaments into account. In Figure 1 the boundaries of the saturated regions are given as a function of the normalized applied field: $B^{*}=4 \pi \alpha t /\left(\mu_{0} \eta j_{c} L_{p}\right)$ and for $a / L_{p}=10^{-1}$ and $10^{-2}$. The only important parameter is the twistlength (for $\eta=0.5$ and $\sigma$ up to $10^{9}\left(\Omega^{-1} m^{-1}\right)$ ). The saturation in the inner region starts at $B^{*}=1$.

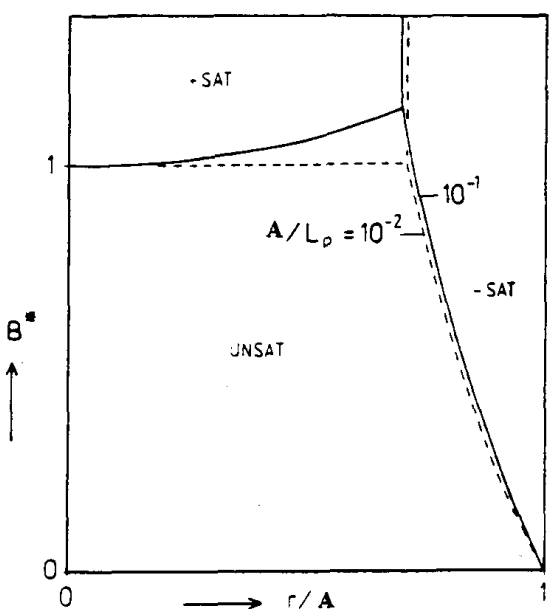

Figure 1: The boundaries of the saturated regions as a function of the normalized applied field $\left(B^{*}=4 \pi \alpha t /\left(\mu_{0} \eta j_{c} L_{p}\right)\right)$.

\section{EXPERIMENTAL SET-UP}

The experimental set-up for measurements on a ring shaped superconducting wire can be sub-divided into a current degradation and a loss measurement with a new calorimetric method.

The sample and the coil are made of a $0.3 \mathrm{~mm}$ NbTi Alsthom wire, with 6534 filaments of $2.3 \mu \mathrm{m}$ diameter and a twistpitch of $3 \mathrm{~mm}$, in a copper matrix. The sample is bifilairly wound on a cylindrical sample holder with an inner diameter of $34 \mathrm{~mm}$. The hight of the sample is $10 \mathrm{~mm}$ and there are 2 layers. The sample length is $5.7 \mathrm{~m}$ and it is 52 times wound around the sample holder. The sample is placed in a DC-coil with a field homogenity of $1 \%$ in the sample volume and a maximum field of $3 T$. The plane of the sample is parallel to the plane of the DC-coil. Perpendicular to this plane, we put an AC-coil inside the sample with a maximum field of a few tenth of a Tesla. (The value depending on the frequency, for example $0.1 \mathrm{~T}$ at $320 \mathrm{~Hz}$.) The dimensions of the coils are chosen in a way, that the DC-coil fits in an available dipole magnet, which can be used to generate a homogeneous AC-field at the sample. Losses occuring in 


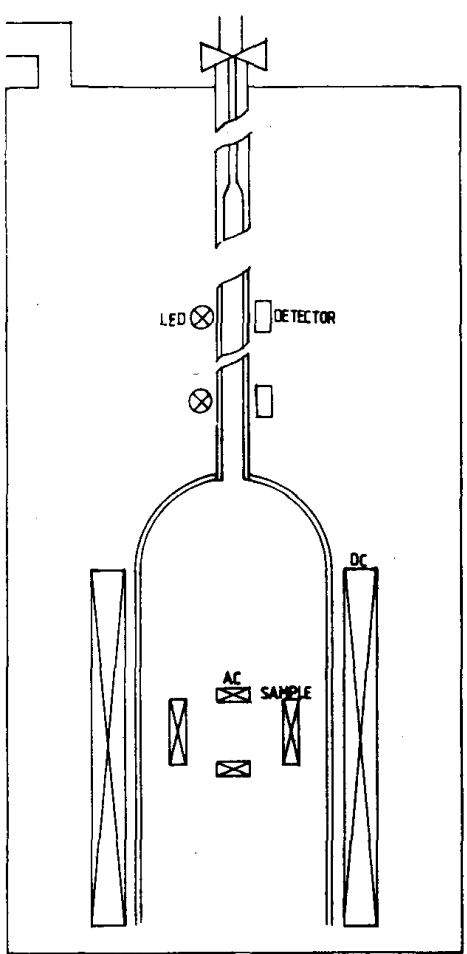

Figure 2: Schematic view of the experimental set-up, showing the configuration of the coils and the calorimeter.

the AC-coil inside the sample will be measured together with the sample losses. Figure 2 shows the configuration of the coils and the calorimeter. For the degradation measurements, the voltage drop across the sample is measured as a function of the transport current and the DC- and AC-fields. The voltage signal will be influenced by the AC-current leads, which introduce a signal on the voltage contacts. This signal is compensated by means of a Rogowski-coil. The remaining voltage signal is further improved by frequency filtering.

There are several methods for calorimetric measurement of AC-losses in superconducting samples. The most well-known method is the boil-off gas measurement with a mass-flow meter. $A$ mass flow meter is very usefull in the range 3-300 $\mathrm{mW}$ with an accuracy of $5 \%$. However, in the lower part of this region the measurements are very difficult and time consuming. As we expect the losses to be in the region around $1 \mathrm{~mW}$ we have to look for another method.

In Kuroda's approach [7] the boil-off gas is collected in a container below the liquid He-surface. Therefore, it is not required to wajt for a static state to re-establish before measuring. Furthermore, there is no heat conduction into the cryostat along the gas-flow tube. The hight of the gas column is measured by eye, the collected gas can be released by pulling out a plug. For this measurement the use of a glass cryostat is, of course, necessary. This is a major drawback. The method has a reported accuracy of $3 \%$ in the range $3-170 \mathrm{~mW}$.

Eikelboom [8] describes a method based on Kuroda's system. He, however, detects the He-gas level in the gas column electronically and removes the gas from the container by rotating it by means of an electromagnet. Eikelboom reports an accuracy of $3 \%$ for the range $1-100 \mathrm{~mW}$.

The method of Kuroda in combination with some of the im- provements of Eikelboom forms the basis for our set-up. We choose to use a container consisting of one part without any moving or rotating parts in liquid He. The sample housing has an inner diameter of $38 \mathrm{~mm}$ and is made of glass fiber with epoxy. The He-level is detected electronically. Instead of a plug inside the cryostat we eventually choose to use a plug at room temperature to reduce the He-leakage we found in a preliminary set-up with a plug below the He-surface.

The calorimeter can be placed inside the DC-coil covering the sample and AC-coil. The boil-off gas from the sample is gathered in the calorimeter and the glass tube on top of it. The time it takes the level to drop from the upper detector to the lower one is a measure for the dissipated power in the sample. The connection between the plug, a normal glass crane, at room temperature and the calorimeter below the liquid He-level is made by a glass tube. To prevent oscillations in the tube, the diameter has to be smaller than $1 \mathrm{~mm}$ [9]. A small diameter also limits the power leakage from room temperature into the measuring system and the influence off pressure variations due to redistribution of temperature is smaller. Therefore, the diameter of the upper part has to be as small as possible.

A smaller diameter of the lower part increases the level-dropspeed and thus increases the accuracy of the level measurement at one detector. If the volume in between the level measuring points decreases, it will be better suited for smaller loss powers.

The signal from the GaAs emitting diode and $\mathrm{Si}$ photodiode combination is filtered with a low-pass filter at $1 \mathrm{~Hz}$. A DCvoltage is substracted in order to have a good visible transition between the tube's filled and empty state. This is required as the time difference between the transitions is measured on an oscilloscope.

It is important, that there is no pressure variation in the cryostat. A pressure regulation circuit is used, using water columns, that keeps the cryostat at approximately $40 \mathrm{~cm}$ water pressure above atmospheric pressure to avoid the influence of pressure variations due to the Helium recover-system.

\section{EXPERIMENTAL RESULTS}

Our first aim is to measure current degradation. Important are the mean value of the voltage drop across the ring shaped sample for V-I curve measurements, but also the form of the voltage, especially the double frequency component. If the wire is nearly saturated as a result of the transport current, then by applying an extra $\mathrm{AC}$-field the wire will partly saturate. Since the field is not the same for the whole wire, there still will be unsaturated parts. If the transport current increases, the saturated part, when applying an $\mathrm{AC}$-field, will increase, giving a rise in voltage. As the direction of the AC-field is not important, the effect occurs at the double frequency of the AC-field. Figure 3 shows the the double frequency component in the measured voltage as function of the applied AC-field for several values of the transport current. We use an AC-field of $250 \mathrm{~Hz}$ and a maximum amplitude of $10 \mathrm{mT}$. The DC-field of $1 T$ is only applied to decrease the critical current. The Lissajous figures show an increase of the double frequency component when the current approaches the critical current. In this case, a bandpass filter at $500 \mathrm{~Hz}$ and $Q=10$ is used. Because of the signal filtering the figures are somewhat smoothened. Especially at $125 \mathrm{~A}$ the edges at the lower end are much sharper. This effect is only clearly observed for frequencies above $25 \mathrm{~Hz}$. Also, the amplitude of the AC-field has its influence on the results.

Figure 4 shows the effect of different frequencies and amplitudes on the V-I curve. For these measurement the mean value of the voltage is required and we filter with a low pass filter at 10 


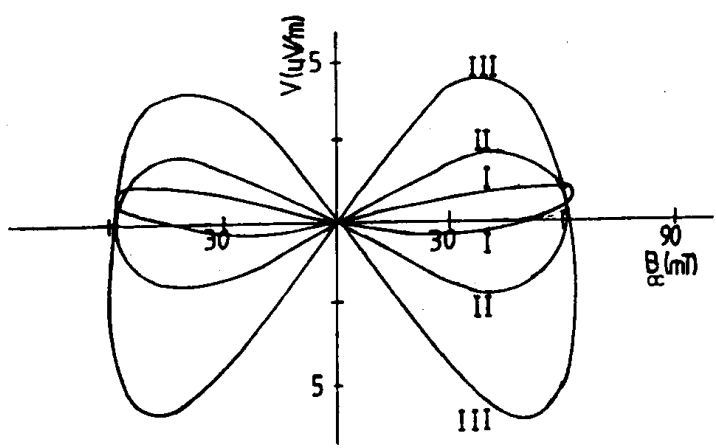

Figure 3: Double frequency component in the measured voltage as function of the applied $\mathrm{AC}$-field for three values of the transport current $I_{t}:$ I) $I_{t}=0 \mathrm{~A}$; II) $I_{t}=100 \mathrm{~A}$; III) $I_{t}=125$ A.

$H z$. The transport current reduces due to an increase in either amplitude or frequency of the applied AC-field. At constant amplitude, the voltage at a certain transport current is linear with frequency up to about the current causing a non-zero voltage in zero AC-field. At constant frequency there is not such a linear relation for the field amplitudes. The voltage increase is of higher order than first in amplitude dependance.

Until now, the loss measurements have been restricted to cali-
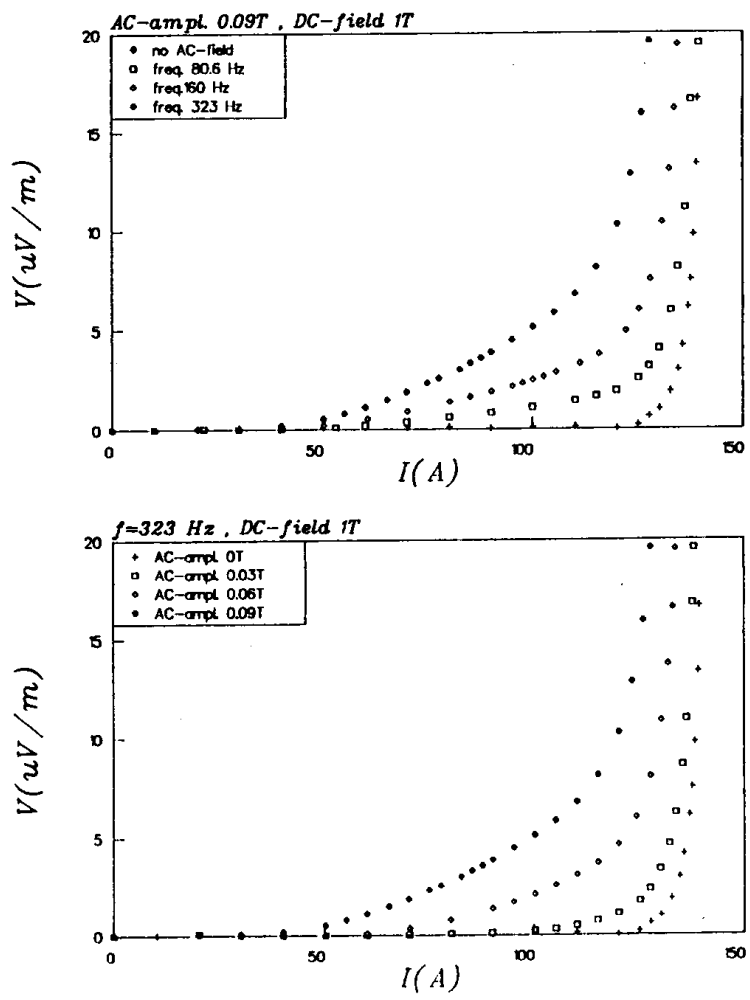

Figure 4: The V-I characteristic of the sample in a perpendicular DC-field of $1 T$, at constant amplitude and varying frequency of the $\mathrm{AC}$-field (upper figure) and vice versa (lower figure). brating the apparatus as well as optimizing the set-up. Most of our efforts in optimizing resulted in adjustments, leading to the set-up described above. The most promising result is found for a measuring tube with upper diameter $1.2 \mathrm{~mm}$ and lower diameter $5 \mathrm{~mm}$ and a volume in between the detectors of $1 \mathrm{~cm}^{3}$. There is an accuracy of $3 \%$ at $8 \mathrm{~mW}$, going up to $4 \%$ at $4 \mathrm{~mW}$ and $8 \%$ at $2 \mathrm{~mW}$. This tube did show some slight oscillations and that is why it is expected to find better results for a tube with smaller upper and lower diameter. These measurements still have to be performed.

\section{CONCLUSIONS}

The stationary solution for the ring shaped wire shows that $\left|\dot{B}_{\| w}^{A}\right|<\eta j_{c} L_{p} /\left(4 \pi R_{0}^{2} \sigma_{\perp}\right)$ for the centre of the wire to be unsaturated. The analysis of the transient response of an infinitely long wire in a parallel applied field shows that $\left|B_{\| w}^{A}\right|<\mu_{0} \eta j_{c} L_{p} /(4 \pi)$ for the centre of the wire to be unsaturated. The measurement of the V-I characteristic of the ring shaped wire gives a V-I curve which is linear dependent on the frequency of the applied field but non linear dependent on the amplitude. This means that the stationary analysis is not complete and transient effects must be considered. The applied time dependent field in the experiment is sinussoidal, while in the theoretical analysis the time dependency is linear. The theoretical description should be extended to incorporate these harmonic fields.

\section{REFERENCES}

[1] E.M.J. Niessen, L.J.M. van de Klundert, R.M.J. van Damme, F.P.H. van Beckum, P.J. Zandbergen, "Coupling losses in superconducting, torus shaped wires due to applied magnetic field changes", unpublished.

[2] W.J. Carr, jr., "Electromagnetic theory for filamentary superconductors", Phys. Rev., Vol. B11, pp. 1547-1554, 1975.

[3] H. London, "Alternating current losses in superconductors of the second kind", Phys. Lett., Vol 6, pp. 162-165, 1963.

[4] C.P. Bean, "Magnetization of hard superconductors", Phys. Rev. Lett., Vol. 8, pp. 250-253, 1962.

[5] B. Turck, "Courants de circulation et pertes dans les composites supraconducteurs soumis a une induction longitudinale variable", Revue de physiquee appliquée, page 369, May 1976.

[6] G. Ries and K.P. Jüngst, "Filament coupling in multifilamentary superconductors in pulsed longitudinal fields", Cryogenics, march 1976.

[7] K. Kuroda, "Modified boil-off method for measuring AC-losses of superconducting composites", Cryogenics, Oct. 1986.

[8] J.A. Eikelboom, "Apparatus for calorimetric measurement of a.c. losses in superconductors", Cryogenics, Vol. 31, May 1991.

[9] T. von Hoffman, U. Lienert, H. Quack, "Experiments on thermally driven gas oscillations", Cryogenics, August 1973. 